\title{
Effect Modification of Multimorbidity on the Association Between Regularity of General Practitioner Contacts and Potentially Avoidable Hospitalisations
}

\author{
Ninh Thi Ha, MHSM ${ }^{7}$ (D), Cameron Wright, $\mathrm{MSc}^{1,2}$, David Youens, $\mathrm{BHS}^{7}$, \\ David B. Preen, $P h D^{3}$, and Rachael Moorin, $P h D^{1,4}$
}

\begin{abstract}
${ }^{1}$ Health Systems and Health Economics, School of Public Health Curtin University, Perth, Western Australia, Australia; ${ }^{2}$ School of Medicine, College of Health \& Medicine, Faculty of HealthUniversity of Tasmania, Hobart, Tasmania, Australia; ${ }^{3}$ Centre for Health Services Research, School of Population and Global HealthThe University of Western Australia, Crawley, WA, Australia; ${ }^{4}$ School of Population and Global HealthThe University of Western Australia, Crawley, WA, Australia.
\end{abstract}

BACKGROUND: Scheduled regular contact with the general practitioner (GP) may lower the risk of potentially avoidable hospitalisations (PAHs). Despite the high prevalence of multimorbidity, little is known about its effect on the relationship between regularity of GP contact and PAHs.

OBJECTIVE: To investigate potential effect modification of multimorbidity on the relationship between regularity of GP contact and probability of PAHs.

DESIGN: A retrospective, cross-sectional study.

PARTICIPANTS: 229,964 individuals aged 45 years and older from the 45 and Up Study in New South Wales, Australia, from 2009 to 2015.

MAIN MEASURES: The main exposure was regularity of GP contact (capturing dispersion of GP contacts); the outcomes were PAHs evaluated by unplanned hospitalisations, chronic ambulatory care sensitive condition (ACSC) hospitalisations and unplanned chronic ACSC hospitalisations. Multivariable logistic regression models and population attributable fractions (PAF) were conducted to identify effect modification of multimorbidity, assessed by Rx-Risk comorbidity score.

KEY RESULTS: Compared with the lowest quintile of regularity, the highest quintile had significantly lower predicted probability of unplanned admission ( -79.9 per 1000 people at risk, 95\% confidence interval (CI) - 85.6; $-74.2)$, chronic ACSC (-6.07 per 1000 people at risk, 95\%CI - 8.07; - 4.08) and unplanned chronic ACSC hospitalisation ( -4.68 per 1000 people at risk, 95\%CI - 6.11; - 3.26). Effect modification of multimorbidity was observed. Specifically, the PAF among people with no multimorbidity indicated that $31.7 \%$ (95\%CI $28.7-34.4 \%)$ of unplanned, 36.4\% (95\%CI 25.1-45.9\%) of chronic ACSC and $48.9 \%$ (95\%CI 32.9-61.1\%) of unplanned chronic ACSC hospitalisation would be reduced by a shift to the highest quintile of regularity. However, among people with $10+$ morbidities, the proportional reduction was only $5.2 \%$ (95\%CI 3.8-6.5\%), 9.0\% (95\%CI 0.5-16.8\%) and $17.8 \%$ (95\%CI 5.4-28.5\%), respectively.

Electronic supplementary material The online version of this article (https://doi.org/10.1007/s11606-020-05699-0) contains supplementary material. which is available to authorized users.

Received July 10, 2019

Revised December 18, 2019

Accepted February 3, 2020

Published online February 24, 2020
CONCLUSIONS: Weakening of the association between regularity and PAHs with increasing levels of multimorbidity suggests a need to improve primary care support to prevent PAHs for patients with multimorbidity.

KEY WORDS: regularity of primary care; avoidable hospitalisation; multimorbidity.

J Gen Intern Med 35(5): 1504-15

DOI: $10.1007 / \mathrm{s} 11606-020-05699-0$

(c) Society of General Internal Medicine 2020

\section{INTRODUCTION}

Multimorbidity is the coexistence of two or more conditions in an individual. ${ }^{1}$ Patients with multimorbidity often require intensive treatment with involvement of multiple health care providers $^{1,2}$ and have a higher risk of iatrogenic harm, due to factors such as drug interactions and suboptimal communication between health care providers. ${ }^{1,2}$ Multimorbidity affects between 55 and $98 \%$ of people aged 65 years and older ${ }^{3}$ and continues to place more pressure on healthcare systems around the world. $1,2,4$

In Australia, the healthcare system has been re-oriented over decades towards strengthening primary care to effectively manage the burden of chronic disease and constrain secondary health system expenditure. ${ }^{5}$ Part of the philosophy behind these changes is that timely and effective treatment and management in the primary care setting can reduce potentially avoidable hospitalisations (PAH). ${ }^{6,7}$

Various aspects of primary health care utilisation can be measured using a variety of tools such as frequency of GP contacts, continuity of provider and regularity of GP contacts. $^{8-12}$ Regularity of GP contacts captures the dispersion of GP contacts, i.e. the extent of the variation in time intervals between contacts with a GP. It is particularly important in the context of high burden of chronic disease as it provides a proxy measure of proactive and planned primary care, which is a main feature of the chronic care model. Studies have found that higher regularity of GP contacts is associated with lower risk of hospitalisation ${ }^{13,14}$ and costs of health care. ${ }^{15}$ Gibson 
et al. ${ }^{16,17}$ found that the Enhanced Primary Care incentives in Australia increased regularity of GP contact with no effect on the number of GP contacts. Regularity has therefore been suggested as a suitable target for health policy interventions aiming at reducing avoidable hospitalisations and controlling healthcare resource use. ${ }^{12,16}$

Although literature highlights the benefit of regular contacts with GPs among certain chronic conditions, limited evidence exists on whether the effect of regularity on hospitalisation may be modified by multimorbidity. Given the high burden of multimorbidity, a better understanding of how multimorbidity may modify the relationship between regularity of GP contact and the risk of hospitalisation would inform whether current primary healthcare delivery models need to be reorganised for people with certain levels of multimorbidity. The aim of this study was to identify the role of multimorbidity in modifying the relationship between regularity of GP contacts and the risk of PAH.

\section{METHODS}

This was a retrospective, longitudinal study using selfreported survey data linked with routinely collected administrative health data from 1 July 2009 to 30 June 2015. Reporting follows the Reporting of studies Conducted using Observational Routinely collected health Data (RECORD) guidelines. ${ }^{18}$

\section{Data Sources}

The study used both self-reported and routinely collected administrative health data linked at the person level from the Sax Institute's 45 and Up Study. ${ }^{19}$

The 45 and Up Study is a longitudinal study of 267,153 participants, aged 45 years and over in the state of New South Wales (NSW), the most populous state located in south-east Australia. Prospective participants were randomly sampled from the Australian Government Department of Human Services (DHS), formerly Medicare Australia, enrolment database and recruited from January 2006 to December 2009. The study methods are described in detail elsewhere. ${ }^{19}$ Briefly, participants completed a baseline health and lifestyle questionnaire at the time they joined the cohort and consented to follow-up and linkage to routine health databases. The overall response rate was $18 \%$ after the first year of recruitment. ${ }^{19}$

The data sources linked and utilised in this study included (i) the 45 and Up Study baseline questionnaire (https://www. saxinstitute.org.au/our-work/45-up-study/); (ii) the NSW Admitted Patient Data Collection (APDC) which provided all discharges from public and private hospitals in NSW (20052017); (iii) the Pharmaceutical Benefits Schedule (PBS) which provided information on dispensed subsidised prescription medicines (2005-2017); (iv) the Medicare Benefits Schedule (MBS) which provided records for all claims for medical and diagnostic services through Medicare, Australia's universal health insurance schedule (2005-2017); and (v) the NSW Register of Births Deaths and Marriages (RBDM) (20062017). The linkage of APDC and RBDM to the survey data was conducted by the NSW Centre for Health Record Linkage. ${ }^{20}$ MBS and PBS data were linked by the Sax Institute using a unique identifier provided by the Department of $\mathrm{Hu}-$ man Services. Quality assurance of the data linkage method showed false-positive and false-negative rates of $<0.5$ and $<$ $0.1 \%$, respectively. ${ }^{20,21}$

Institutional ethics committee approval was obtained from Curtin University Human Research Ethics Committee (RD-42-14) and the NSW Population and Health Services Research Ethics Committee (HREC/17/CIPHS/ 37). Consent was given by all participants in the 45 and Up Study for their information to be used in approved studies, and for follow-up and data linkage. The conduct of the 45 and Up Study was approved by the University of NSW Human Research Ethics Committee.

\section{Study Timeline}

The study was structured into three periods: baseline $(1 \mathrm{Ju}-$ ly 2005 to 30 June 2009); follow-up period 1 (F1, 1 July 2009 to 30 June 2012) and follow-up 2 (F2) from 1 July 2012 to 30 June 2015 (see Appendix 1). By doing this, any effects of the previous exposure in F1 and baseline on the outcome in F2 were controlled in the model to isolate the immediate effect of regularity in the F2.

\section{Cohort}

All participants of the 45 and Up Study recruited prior to 1 January 2008 who were still alive on 1 July 2009 were eligible for the study. Participants with potential linkage errors, those who died before 1 July $2012(n=13,653)$ and those with less than three GP contacts in any 3-year follow-up period $(n=$ 23,536 ) were excluded, as this was the minimum number of contacts required to calculate the regularity and continuity variables.

\section{Outcome Variables}

The main outcome measure of this study was potentially avoidable hospitalisation observed during the period F2. Despite its wide use, the definition of PAH is not standardised. ${ }^{22}$, ${ }^{23}$ Thus, this study evaluated PAH using three measures: unplanned hospitalisation, hospitalisation for chronic ACSC and unplanned chronic ACSC corresponding to a broad, restricted and highly restricted form, respectively. The dependent variables were binary (yes/no) indicators of three types of hospitalisation events observed during F2.

i. Any unplanned hospitalisation (representing the lowest level of potentially avoidable hospitalisation), identified using the emergency status (urgency of admission) variable in the APDC data; 
ii. Hospitalisation for a chronic ACSC. These included conditions classified as PAH through the provision of appropriate individualised preventative health interventions and early disease management usually delivered in primary care and community-based care settings by the National Health Performance Framework. ${ }^{24,}{ }^{25}$ These hospitalisations were ascertained using the International Statistical Classification of Diseases and Related Health Problems, Tenth Revision; Australian Modification (ICD-10-AM) codes.

iii. Chronic ACSC hospitalisations identified in (ii) and classified as unplanned.

To avoid overestimating outcomes, inter-hospital transfers were counted as a single hospitalisation event. Hospitalisations categorised as type (i), (ii) or (iii) occurring in the first and second time periods were used as covariates in their respective models to adjust for prior history of the outcome of interest.

\section{Regularity of GP Contacts}

GP contact was captured via MBS claims for "Attendances by General Practitioners". ${ }^{26}$ General practitioner in this context refers to physicians only; nurse practitioners, physician assistants and so on are not included. ${ }^{27}$ The MBS contains a series of billable item numbers, primarily used by GPs visited by patients in private primary care clinics. Regularity of GP contact was measured at each time period using the previously reported Modified Regularity Index ${ }^{11}$ as follows. (See Appendix 2 for further details.)

$R_{c v}=1 /(1+c v($ days $))$

where $\mathrm{cv}$ is coefficient of variation.

The index captures dispersion of GP contacts based on the coefficient of variation in the time intervals (days) between GP contacts within an ascertainment period (3-year period in this study) including the time interval from the beginning to the first GP contact and from the last GP contact in the period to the end of the period. This score was separated into regularity quintiles, from least to most regular, using the range of scores observed in the study population.

\section{Multimorbidity}

Multimorbidity was captured using the Rx-Risk index. The index is a count indicating the number of comorbidities a participant has, based on prescribing data, and was ascertained using four and a half and 5-year look back of the PBS data from the date of the start of F1 and F2, respectively. ${ }^{28}$

\section{Other Covariates}

Other GP utilisation covariates were captured in each time period including the number of chronic disease and mental health-related MBS-funded primary care services in each time period; continuity of provider using both the Modified
Modified Continuity Index (MMCI) ${ }^{29}$ and the Usual Provider of Care Index (UPC) ${ }^{8,} 30$ (see Appendix 2 for formulae). The de-identified provider number in the MBS data were used to distinguish different GPs to calculate both UPC and MMCI. Briefly, the UPC indicates the proportion of visits by the main GP for each individual, while the MMCI indicates the degree of spread of visits across providers for each individual. Both indices were reported using the following categories: low (< $50 \%)$, moderate (50-75\%), high (76-90\%) and very high (91$100 \%)$. The frequency of GP contacts was ascertained for each time period as a count of the number of days each person had a GP contact.

Self-report information on key potential confounders were obtained from the 45 and Up Study baseline questionnaire including: age; sex; marital status; born in Australia (yes/no); Indigenous status; current housing; household income; education level; smoking status; alcohol use; physical activity ${ }^{31}$; time spent sitting; body mass index; psychological distress ${ }^{32}$; level of limitation reported (based on the 36 Item Short Form survey (SF-36) ${ }^{33}$; social support ${ }^{34}$; self-rated overall health and quality of life; and self-reported previous diagnosis for chronic conditions (see Appendix 3 for categories).

Socio-economic status and accessibility to services were derived from the postcode of residence at time of recruitment and reported using the Socio-Economic Index for Areas Index of Relative Socio-economic Disadvantage ${ }^{35}$ and the Accessibility/Remoteness Index of Australia. ${ }^{36}$ Use of specialist physician services, Medicare-funded chronic disease management items and mental health-related services were captured using MBS claims data for each time period. A binary variable was used to capture if the participant died during F2. Person-time at risk of the outcome event, defined as the number of days alive and not in hospital in F2, was also included as a covariate in the regression models. Risk of hospitalisation attributable to history of admission with comorbidity was captured using the Multipurpose Australian Comorbidity Scoring System (MACSS),${ }^{37}$ defined as the sum of comorbidities reported on APDC records at 5 years ascertained prior to the start of each time period.

\section{Statistical Analysis}

Descriptive statistics were generated for socio-demographics and health service use across quintiles of regularity. The effect modification of multimorbidity on the relationship between regularity of GP contact and the probability of PAH was examined using the interaction term in multivariable logistic regressions incorporating robust standard errors and postestimation average marginal effect. Wald tests and likelihood ratio tests were performed to confirm the interaction ${ }^{38}$ and the effect modification of multimorbidity. ${ }^{39}$ The differences in probability of $\mathrm{PAH}$ between higher regularity quintiles versus the lowest regularity quintile were computed across values of multimorbidity to indicate the effect medication of multimorbidity. 
Population attributable fractions (PAF) and population unattributable fractions (PUF) were calculated using the user written STATA package "punaf". ${ }^{40}$ The PAF was used to determine proportional reduction of specified PAH potentially attributable to a shift to the highest quintile of regularity of GP contact in a hypothetical world for all the population. The PUF indicated the proportion of PAH that would potentially remain under the hypothetical world. The analyses were conducted for whole study population and separately for the population with no Rx risk, 1-5 Rx risk, 6-10 Rx risk and >10 Rx risk across specified types of hospitalisation to indicate variation in effect of regularity across different levels of multimorbidity.

All logistic regression models controlled for all baseline characteristics, time varying GP utilisation (baseline, F1 and F2), number of specialist visits (baseline and F1), risk of hospitalisation attributable to comorbidity (baseline and entry to F1), Rx risk (baseline and entry to F1 and F2), and history of unplanned/chronic ACSC/ unplanned chronic ACSC.

Analyses were undertaken using Stata SE Version 14.2. ${ }^{41}$

\section{RESULTS}

\section{Cohort Characteristics}

Overall, 229,964 individuals from the 45 and Up Study met our inclusion criteria. The median age at baseline was 61 years (IQR, 53-69), with 56\% female and 98\% non-Indigenous. Regularity of GP contacts had a mean of 0.218 (SD 0.05) for the baseline period. Similar distributions were observed in follow-up period 1 (mean of 0.219 (SD 0.049)) and followup period 2 (mean of 0.217 (SD 0.05)).

Table 1 shows that the distribution of baseline characteristics was similar across regularity quintiles, except for levels of limitation with a slightly higher proportion of severe limitation among those with higher regularity. Further cohort characteristics are presented in Appendix 4.

Table 2 shows the distribution of multimorbidity and health service utilisation across regularity quintiles at the second follow-up (F2). High multimorbidity including 6-10 conditions and $>10$ conditions was more likely among those with higher quintiles of regularity. Considering primary care, individuals in the higher regularity quintiles were also more likely to have very high UPC and MMCI compared with those in the lower regularity quintile. Twenty-five percent had an unplanned hospitalisation, $1.9 \%$ had a chronic ACSC hospitalisation and $0.9 \%$ had an unplanned chronic ACSC during the second follow-up period.

\section{Associations Between Regularity and Specified Types of Hospitalisations}

Table 3 shows that after adjusting for demographic and clinical characteristics, regularity of GP contacts was significantly associated with reduction in probability of having unplanned, chronic ACSC and unplanned chronic ACSC hospitalisations.
Significant associations were observed across different quintiles of regularity. The highest coefficient was observed in the highest quintile of regularity $-0.69(95 \% \mathrm{CI}-0.75 ;-0.63),-$ $0.71(95 \% \mathrm{CI}-0.92 ;-0.50)$ and $-1.05(95 \% \mathrm{CI}-1.39$; 0.72 ) for unplanned, chronic ACSC and unplanned chronic ACSC hospitalisations, respectively.

Compared with the lowest quintile of regularity, people in higher regularity quintiles had a significantly lower predicted probability of unplanned hospital admission with -19.5 , $37.2,-46.2$ and -79.9 per 1000 population for quintiles 2 to 5 , respectively (Table 4B). For chronic ACSC admission and unplanned chronic ACSC admission, a significant association between regularity quintiles and hospitalisation was only observed from moderate to highest regularity quintile relative to the lowest regularity quintile (Table 4B). A pairwise contrast between each regularity quintile and its immediate lower counterpart in terms of predicted probability of unplanned hospitalisation was significant across all pairs of regularity quintile (Table 4C). However, for chronic ACSC and unplanned chronic ACSC hospitalisation, the pairwise contrast was only significant between the highest and high regularity quintile (Table 4C).

\section{Effect Modification of Multimorbidity}

Figure 1 shows that across the specified types of hospitalisation (all unplanned, chronic ACSC and unplanned chronic ACSC), the association between regularity quintile and probability of having the hospitalisation was modified by level of multimorbidity. Among those with no morbidity, higher regularity quintiles were significantly associated with lower probability of having an unplanned hospitalisation. However, when multimorbidity levels were greater than 10 , no association was observed between higher regularity quintiles and the probability of having the unplanned hospitalisation, except for the highest regularity quintile.

The effect modification of multimorbidity was more apparent for chronic ACSC and unplanned chronic ACSC hospitalisations (Fig. 1). No association between regularity quintiles and the probability of specified hospitalisation was observed when level of multimorbidity was greater than 7 for chronic ACSC hospitalisations and greater than 9 for unplanned chronic ACSC hospitalisations (Appendix 5).

Assessment of the PAF is shown in Fig. 2. The probability of unplanned, chronic ACSC and unplanned chronic ACSC potentially prevented if the cohort all achieved the highest quintile of regularity was $17.2 \%$ (95\% CI $15.9-18.5 \%$ ), $19.5 \%$ (95\% CI $14.0-24.7 \%$ ) and 28.9 (95\% CI $21.3-$ $35.7 \%$ ), respectively. However, the preventive fraction of the hospitalisation attributable to a move to the highest quintile of regularity was lower with increased multimorbidity. Among those with no multimorbidity, the preventive fraction attributable to the highest regularity was $31.7 \%$ (95\%CI, 28.7$34.5 \%$ ) of unplanned, $36.4 \%$ (95\% CI, 25.1-45.9\%) of chronic ACSC and $48.9 \%$ (95\% CI, 32.9-61.1\%) of unplanned chronic ACSC hospitalisation. In contrast, among those with 10 or 
Table 1 Selected Baseline Characteristics of the Study Population Across Regularity Quintile in the Second Follow-up Period

\begin{tabular}{|c|c|c|c|c|c|c|}
\hline \multirow[t]{3}{*}{ Characteristics } & \multicolumn{5}{|c|}{ Regularity quintile in second follow-up period F2 } & \multirow[b]{2}{*}{$\begin{array}{l}\text { Total of those with } 3+\text { GP } \\
\text { contacts }(N=229,964)\end{array}$} \\
\hline & \multirow{2}{*}{$\begin{array}{l}\text { Lowest }(N= \\
42,264) \\
n(\%)\end{array}$} & \multirow{2}{*}{$\begin{array}{l}\text { Low }(N= \\
47,497) \\
n(\%)\end{array}$} & \multirow{2}{*}{$\begin{array}{l}\text { Moderate }(N= \\
48,080) \\
n(\%)\end{array}$} & \multirow{2}{*}{$\begin{array}{l}\text { High }(N= \\
47,532) \\
n(\%)\end{array}$} & \multirow{2}{*}{$\begin{array}{l}\text { Highest }(N= \\
44,591) \\
n(\%)\end{array}$} & \\
\hline & & & & & & $n(\%)$ \\
\hline $\begin{array}{l}\text { Age at recruit year } \\
\text { (median }[\mathrm{IQR}])\end{array}$ & $\begin{array}{l}58[51.5- \\
66.8]\end{array}$ & $\begin{array}{l}59.8[52.8- \\
68.2]\end{array}$ & $61.1[53.8-69.3]$ & $\begin{array}{l}61.7[54.3- \\
70.1]\end{array}$ & $\begin{array}{l}61.2[54.0- \\
70.0]\end{array}$ & $60.5[53.2-69.0]$ \\
\hline Sex & $22,367(52.9)$ & $\begin{array}{l}26,386 \\
(55.6)\end{array}$ & $27,414(57.0)$ & $\begin{array}{l}27,122 \\
(57.1)\end{array}$ & $24,465(54.9)$ & $127,754(55.6)$ \\
\hline $\begin{array}{l}\text { Indigenous status } \\
\text { Not indigenous }\end{array}$ & $41,218(97.5)$ & $\begin{array}{l}46,352 \\
(97.6)\end{array}$ & $46,953(97.7)$ & $\begin{array}{l}46,406 \\
(97.6)\end{array}$ & $43,492(97.5)$ & $224,421(97.6)$ \\
\hline $\begin{array}{l}\text { Indigenous } \\
\text { Not reported }\end{array}$ & $\begin{array}{l}324(0.8) \\
722(1.7)\end{array}$ & $\begin{array}{l}358(0.8) \\
787(1.7)\end{array}$ & $\begin{array}{l}348(0.7) \\
779(1.6)\end{array}$ & $\begin{array}{l}347(0.7) \\
779(1.6)\end{array}$ & $\begin{array}{l}324(0.7) \\
775(1.7)\end{array}$ & $\begin{array}{l}1701(0.7) \\
3842(1.7)\end{array}$ \\
\hline \multicolumn{7}{|l|}{ Born in Australia } \\
\hline $\begin{array}{l}\text { Yes } \\
\text { Marital status }\end{array}$ & $30,139(71.3)$ & $35,027(73.7)$ & $36,299(75.5)$ & $36,399(76.6)$ & $34,701(77.8)$ & $172,565(75)$ \\
\hline $\begin{array}{l}\text { Single, widowed, } \\
\text { divorced, separated }\end{array}$ & $10,651(25.2)$ & $11,317(23.8)$ & $11,284(23.5)$ & $11,230(23.6)$ & $11,127(25.0)$ & $55,609(24.2)$ \\
\hline $\begin{array}{l}\text { Married/living with a } \\
\text { partner }\end{array}$ & $31,295(74)$ & $35,908(75.6)$ & $36,559(76)$ & $36,043(75.8)$ & $33,206(74.5)$ & $173,011(75.2)$ \\
\hline \multicolumn{7}{|l|}{ SEIFA $* *$} \\
\hline Least disadvantaged & $8561(20.3)$ & $9747(20.5)$ & $10,044(20.9)$ & $9826(20.7)$ & $9305(20.9)$ & $47,483(20.6)$ \\
\hline Disadvantaged & $6962(16.5)$ & $7997(16.8)$ & $8108(16.9)$ & $8047(16.9)$ & $7596(17)$ & $38,710(16.8)$ \\
\hline Moderate disadvantage & $8032(19)$ & 9015 (19) & $9004(18.7)$ & $8844(18.6)$ & $8187(18.4)$ & $43,082(18.7)$ \\
\hline High disadvantage & $8941(21.2)$ & $10,072(21.2)$ & $9980(20.8)$ & $9845(20.7)$ & $9236(20.7)$ & $48,074(20.9)$ \\
\hline Highest disadvantage & $8673(20.5)$ & $9429(19.9)$ & $9643(20.1)$ & $9770(20.6)$ & $9112(20.4)$ & $46,627(20.3)$ \\
\hline Not reported & $1095(2.6)$ & $1237(2.6)$ & $1301(2.7)$ & $1200(2.5)$ & $1155(2.6)$ & $5988(2.6)$ \\
\hline \multicolumn{7}{|l|}{ Accessibility } \\
\hline Highly accessible & $22,450(53.1)$ & $\begin{array}{l}25,342 \\
(53.4)\end{array}$ & $25,527(53.1)$ & $\begin{array}{l}24,556 \\
(51.7)\end{array}$ & $22,533(50.5)$ & $120,408(52.4)$ \\
\hline Accessible & $14,175(33.5)$ & $\begin{array}{l}16,262 \\
(34.2)\end{array}$ & $16,612(34.6)$ & $\begin{array}{l}16,822 \\
(35.4)\end{array}$ & $16,151(36.2)$ & $80,022(34.8)$ \\
\hline Moderately & $4439(10.5)$ & $4591(9.7)$ & $4646(9.7)$ & $4842(10.2)$ & $4720(10.6)$ & $23,238(10.1)$ \\
\hline Remote/very remote & $452(1.1)$ & $441(0.9)$ & $419(0.9)$ & $449(0.9)$ & $349(0.8)$ & $2110(0.9)$ \\
\hline \multirow{2}{*}{\multicolumn{7}{|c|}{ Level of limitation }} \\
\hline & & & & & & \\
\hline No & $13,682(32.4)$ & $\begin{array}{l}14,063 \\
(29.6)\end{array}$ & $13,245(27.5)$ & $\begin{array}{l}12,435 \\
(26.2)\end{array}$ & $11,900(26.7)$ & $65,325(28.4)$ \\
\hline Minor & $13,348(31.6)$ & $\begin{array}{l}15,380 \\
(32.4)\end{array}$ & $15,672(32.6)$ & $\begin{array}{l}15,312 \\
(32.2)\end{array}$ & $14,002(31.4)$ & $73,714(32.1)$ \\
\hline Moderate & $7931(18.8)$ & 9777 (20.6) & $10,537(21.9)$ & $\begin{array}{l}11,020 \\
(23.2)\end{array}$ & $9960(22.3)$ & $49,225(21.4)$ \\
\hline $\begin{array}{l}\text { Severe } \\
\text { Psychological distress }\end{array}$ & $7303(17.3)$ & $8277(17.4)$ & $8626(17.9)$ & $8765(18.4)$ & 8729 (19.6) & $41,700(18.1)$ \\
\hline $\begin{array}{l}\text { Psychological distress } \\
\text { Low }\end{array}$ & $33,406(79)$ & $37,516(79)$ & $38,179(79.4)$ & $\begin{array}{l}37,702 \\
(79.3)\end{array}$ & $35,371(79.3)$ & $182,174(79.2)$ \\
\hline Moderate & $5992(14.2)$ & $6875(14.5)$ & $6803(14.1)$ & $6739(14.2)$ & $6196(13.9)$ & $32,605(14.2)$ \\
\hline High & $2129(5.0)$ & $2251(4.7)$ & $2254(4.7)$ & $2197(4.6)$ & $2163(4.9)$ & $10,994(4.8)$ \\
\hline Very high & $737(1.7)$ & $855(1.8)$ & $844(1.8)$ & 894 (1.9) & 861 (1.9) & $4191(1.8)$ \\
\hline \multicolumn{7}{|l|}{ Self-reported overall health } \\
\hline Excellent & $6902(16.3)$ & $7044(14.8)$ & $6781(14.1)$ & $6285(13.2)$ & $5951(13.3)$ & $32,963(14.3)$ \\
\hline Very good & $15,674(37.1)$ & $\begin{array}{l}17,661 \\
(37.2)\end{array}$ & $17,841(37.1)$ & $\begin{array}{l}17,004 \\
(35.8)\end{array}$ & $15,804(35.4)$ & $83,984(36.5)$ \\
\hline Good & $13,460(31.8)$ & $\begin{array}{l}15,695 \\
(33.0)\end{array}$ & $15,969(33.2)$ & $\begin{array}{l}16,344 \\
(34.4)\end{array}$ & $14,716(33.0)$ & $76,184(33.1)$ \\
\hline Fair & $4127(9.8)$ & $4816(10.1)$ & $5122(10.7)$ & $5456(11.5)$ & $5614(12.6)$ & 25,135 (10.9) \\
\hline Poor & $589(1.4)$ & $655(1.4)$ & $767(1.6)$ & $830(1.7)$ & $1069(2.4)$ & $3910(1.7)$ \\
\hline \multirow{2}{*}{\multicolumn{7}{|c|}{ Self-reported diagnosis of chronic conditions }} \\
\hline & & & & & & \\
\hline Asthma & $4094(9.7)$ & $4989(10.5)$ & $5182(10.8)$ & $5238(11)$ & $4705(10.6)$ & $24,208(10.5)$ \\
\hline Diabetes & $2636(6.2)$ & $3793(8.0)$ & $4472(9.3)$ & $4859(10.2)$ & $4733(10.6)$ & $20,493(8.9)$ \\
\hline Stroke & $956(2.3)$ & $1196(2.5)$ & $1307(2.7)$ & $1462(3.1)$ & $1558(3.5)$ & $6479(2.8)$ \\
\hline Blood clot thrombosis & $1672(4.0)$ & $2058(4.3)$ & $2166(4.5)$ & $2293(4.8)$ & $2313(5.2)$ & $10,502(4.6)$ \\
\hline Heart disease & $3460(8.2)$ & $4749(10)$ & $5457(11.3)$ & $6123(12.9)$ & $6120(13.7)$ & $25,909(11.3)$ \\
\hline Cancer & $13,654(32.3)$ & $\begin{array}{l}16,928 \\
(35.6)\end{array}$ & $17,641(36.7)$ & $\begin{array}{l}17,702 \\
(37.2)\end{array}$ & $15,910(35.7)$ & $81,835(35.6)$ \\
\hline Anxiety OR depression & $6469(15.3)$ & $8012(16.9)$ & $8376(17.4)$ & $8555(18.0)$ & $7726(17.3)$ & $39,138(17)$ \\
\hline High blood pressure & $11,277(26.7)$ & $\begin{array}{l}15,863 \\
(33.4)\end{array}$ & $18,212(37.9)$ & $\begin{array}{l}19,427 \\
(40.9)\end{array}$ & $19,334(43.4)$ & $84,113(36.6)$ \\
\hline
\end{tabular}

Lowest quintile regularity: 0-0.204; low quintile: 0.205-0.217; medium quintile: 0.217-0.227; high quintile: 0.228-0.240; highest quintile: 0.241-1 Data are presented as median (IQR) for continuous measures, and $n(\%)$ for categorical measures

*F2: the second follow-up period; ** SEIFA: Socio-Economic Index for Areas Index of Relative Socio-economic Disadvantage 
Table 2 Distribution of Multimorbidity and Health Service Use Across Regularity Quintile in the Second Follow-up Period (F2)

\begin{tabular}{|c|c|c|c|c|c|c|}
\hline \multirow[t]{3}{*}{ Characteristics } & \multicolumn{5}{|c|}{ Regularity quintile in second follow-up period F2 } & \multirow{2}{*}{$\begin{array}{l}\text { Total of those with 3+ } \\
\text { GP contacts }(N= \\
229,964)\end{array}$} \\
\hline & \multirow{2}{*}{$\begin{array}{l}\begin{array}{l}\text { Lowest } \\
(N=42,264)\end{array} \\
N(\%)\end{array}$} & \multirow{2}{*}{$\begin{array}{l}\text { Low }(N= \\
47,497) \\
N(\%)\end{array}$} & \multirow{2}{*}{$\begin{array}{l}\begin{array}{l}\text { Moderate } \\
(N=48,080)\end{array} \\
N(\%)\end{array}$} & \multirow{2}{*}{$\begin{array}{l}\text { High }(N= \\
47,532) \\
N(\%)\end{array}$} & \multirow{2}{*}{$\begin{array}{l}\begin{array}{l}\text { Highest } \\
(N=44,591)\end{array} \\
N(\%)\end{array}$} & \\
\hline & & & & & & $N(\%)$ \\
\hline \multicolumn{7}{|l|}{$\mathrm{UPC}^{*}$ in $\mathrm{F} 2$} \\
\hline Low & $\begin{array}{l}12,815 \\
(30.3)\end{array}$ & $\begin{array}{l}13,708 \\
(28.9)\end{array}$ & $11,970(24.9)$ & $9872(20.8)$ & $6494(14.6)$ & $54,859(23.9)$ \\
\hline Moderate & $\begin{array}{l}15,808 \\
(37.4)\end{array}$ & $\begin{array}{l}17,521 \\
(36.9)\end{array}$ & $17,501(36.4)$ & $\begin{array}{l}16,829 \\
(35.4)\end{array}$ & $13,604(30.5)$ & $81,263(35.3)$ \\
\hline High & $7637(18.1)$ & $9143(19.2)$ & $10,232(21.3)$ & $\begin{array}{l}11,058 \\
(23.3)\end{array}$ & $11,114(24.9)$ & $49,184(21.4)$ \\
\hline $\begin{array}{l}\text { Very high } \\
\text { MMCI** in F2 }\end{array}$ & $6004(14.2)$ & $7125(15.0)$ & $8377(17.4)$ & $9773(20.6)$ & $13,379(30.0)$ & $44,658(19.4)$ \\
\hline \multicolumn{7}{|l|}{ MMCI** in F2 } \\
\hline $\begin{array}{l}\text { Low } \\
\text { Moderate }\end{array}$ & $6375(15.1)$ & $6561(13.8)$ & $5810(12.1)$ & $5662(11.9)$ & $6047(13.6)$ & $30,455(13.2)$ \\
\hline Moderate & $9720(23.0)$ & $10,551(22.2)$ & $9935(20.7)$ & $9161(19.3)$ & $7147(16.0)$ & $46,514(20.2)$ \\
\hline High MMCI & $12,996(30.7)$ & $15,010(31.6)$ & $15,332(31.9)$ & $14,397(30.3)$ & $11,196(25.1)$ & $68,931(30)$ \\
\hline Very high MMCI & $13,173(31.2)$ & $15,375(32.4)$ & $17,003(35.4)$ & $18,312(38.5)$ & $20,201(45.3)$ & $84,064(36.6)$ \\
\hline \multicolumn{7}{|c|}{ Rx risk multimorbidity ( 5 years prior to $\mathrm{F} 2$ ) } \\
\hline No & $\begin{array}{l}16,008 \\
(37.9)\end{array}$ & $12,842(27)$ & $10,744(22.3)$ & $9656(20.3)$ & $9693(21.7)$ & $58,943(25.6)$ \\
\hline $1-5$ conditions & $\begin{array}{l}18,273 \\
(43.2)\end{array}$ & $\begin{array}{l}22,903 \\
(48.2)\end{array}$ & $23,391(48.7)$ & $\begin{array}{l}22,642 \\
(47.6)\end{array}$ & $20,952(47.0)$ & $108,161(47.0)$ \\
\hline $6-10$ conditions & $7014(16.6)$ & $\begin{array}{l}10,420 \\
(21.9)\end{array}$ & $12,198(25.4)$ & $\begin{array}{l}13,073 \\
(27.5)\end{array}$ & $11,805(26.5)$ & $54,510(23.7)$ \\
\hline$>10$ conditions & $969(2.3)$ & $1332(2.8)$ & $1747(3.6)$ & $2161(4.5)$ & $2141(4.8)$ & $8350(3.6)$ \\
\hline Unplanned hospitalisation F2 & $\begin{array}{l}11,925 \\
(28.2)\end{array}$ & $\begin{array}{l}12,390 \\
(26.1)\end{array}$ & $12,114(25.2)$ & $\begin{array}{l}11,770 \\
(24.8)\end{array}$ & $9489(21.3)$ & $57,688(25.1)$ \\
\hline Chronic ACSC A** $^{* *}$ hospitalisation F2 & $790(1.9)$ & $899(1.9)$ & $917(1.9)$ & $998(2.1)$ & $762(1.7)$ & $4366(1.9)$ \\
\hline $\begin{array}{l}\text { Unplanned chronic ACSC } \\
\text { hospitalisation F2 }\end{array}$ & $433(1.0)$ & $438(0.9)$ & $445(0.9)$ & $504(1.1)$ & $340(0.8)$ & $2160(0.9)$ \\
\hline $\begin{array}{l}\text { MACCS**** (5 years prior to F2) } \\
\text { (median, [IQR }] \text { ) }\end{array}$ & $0[0-2]$ & $0[0-3]$ & $0[0-4]$ & $0[0-4]$ & $0[0-3]$ & $0[0-3]$ \\
\hline $\begin{array}{l}\text { Rx risk multimorbidity (5 years prior } \\
\text { to F2) (median, [IQR]) }\end{array}$ & $2[0-5]$ & $3[0-5]$ & $3[1-6]$ & $4[1-6]$ & $3[1-6]$ & $3[0-6]$ \\
\hline $\begin{array}{l}\text { Number of chronic disease } \\
\text { management GP contacts in F2 } \\
\text { (median, [IQR]) }\end{array}$ & $0[0-2]$ & $0[0-3]$ & $0[0-4]$ & $0[0-4]$ & $0[0-3]$ & $0[0-3]$ \\
\hline $\begin{array}{l}\text { Number of mental health GP contacts } \\
\text { F2 (median, }[\mathrm{IQR}] \text { ) }\end{array}$ & $0[(0-0)]$ & $0[(0-0)]$ & $0[(0-0)]$ & $0[(0-0)]$ & $0[(0-0)]$ & $0[(0-0)]$ \\
\hline $\begin{array}{l}\text { Frequency of GP contacts in F2 } \\
\text { (median, [IQR]) }\end{array}$ & 21 [13-34] & 23 [15-35] & 23 [15-35] & 21 [14-34] & 16 [10-30] & 21 [13-34] \\
\hline
\end{tabular}

Lowest quintile regularity: 0-0.204; low quintile: 0.205-0.217; medium quintile: 0.217-0.227; high quintile: 0.228-0.240; highest quintile: 0.241-1 Data are presented as median (IQR) for continuous measures, and $n$ (\%) for categorical measures

F2: the second follow-up period

*UPC: Usual Provider of Care Index

**MMCI: Modified Modified Continuity Index

***ACSC: Ambulatory care sensitive conditions

****MACCS: Multipurpose Australian Comorbidity Scoring System

more conditions, the proportion of unplanned, chronic ACSC and unplanned ACSC hospitalisation that might be prevented attributable to the highest regularity quintile was only $5.2 \%$ (95\% CI 3.8-6.5\%), 9.0\% (4.8-16.8\%) and $17.8 \%(5.4-$ $28.5 \%)$, respectively.

\section{DISCUSSION}

To our knowledge, this was the first study to examine the effect modification of multimorbidity on the association between GP regularity and the probability of hospitalisation. Our results suggest the existence of effect modification by multimorbidity on the association between regularity of GP con- tacts and hospitalisation. Higher GP contact regularity was significantly associated with a reduction in the probability of each hospitalisation type, similar to that shown in literature. ${ }^{13 \text {, }}$ 14, ${ }^{2}$ However, the reduction diminished with increasing multimorbidity. The effect modification of multimorbidity was most apparent for chronic ACSC and unplanned chronic ACSC hospitalisations.

Our study suggests a considerable difference in the association of regularity between those with no multimorbidity and those with very high multimorbidity. The weaker association of regularity among people with high multimorbidity found in our study highlights the concern of fragmented care for people with multimorbidity. This has been explored through GP- and patient-focused qualitative studies. ${ }^{43-46}$ People with 
Table 3 Association between quintile regularity and hospital outcomes in the second follow-up period (F2)

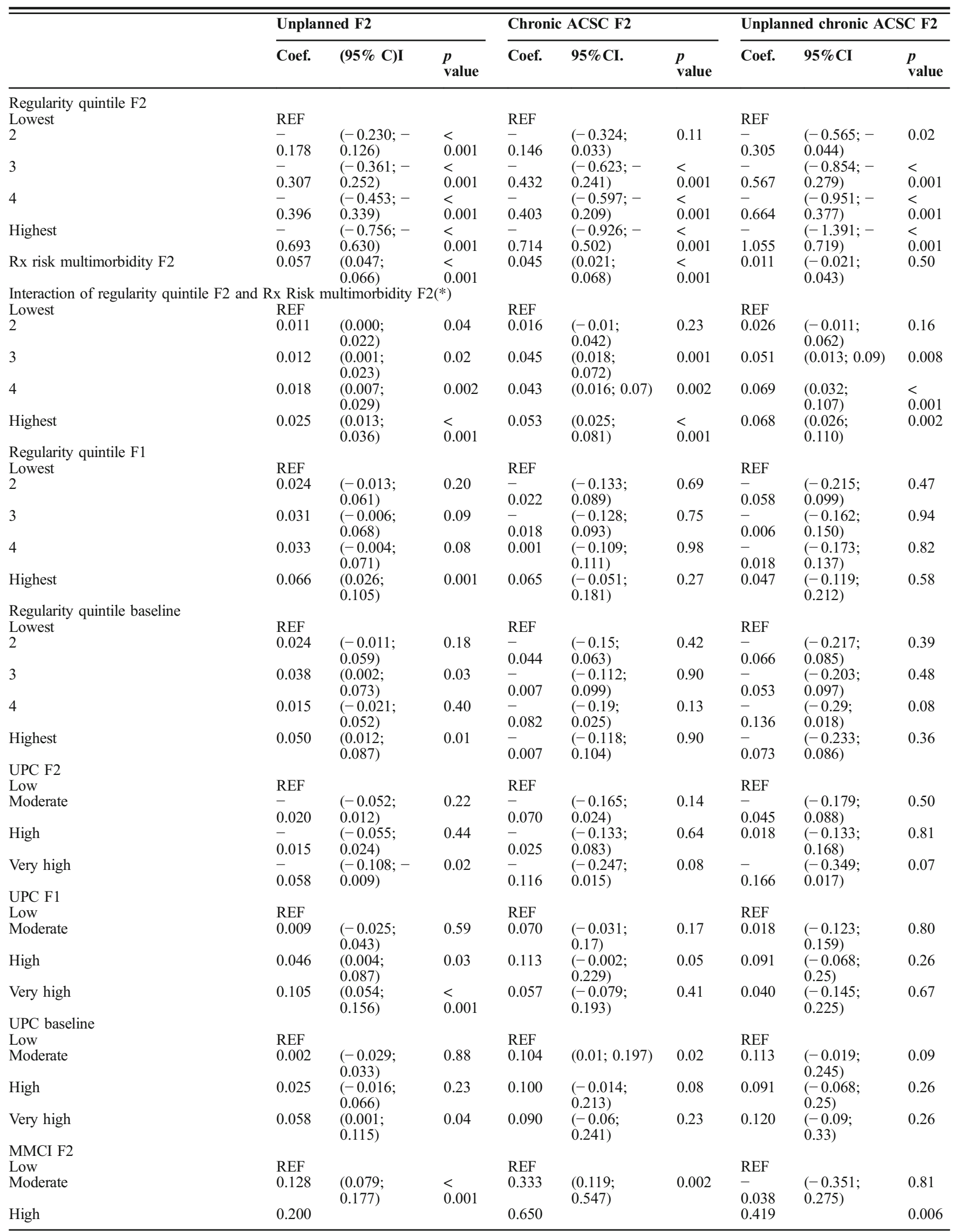


Table 3. (continued)

\begin{tabular}{|c|c|c|c|c|c|c|c|c|c|}
\hline & \multicolumn{3}{|c|}{ Unplanned F2 } & \multicolumn{3}{|c|}{ Chronic ACSC F2 } & \multicolumn{3}{|c|}{ Unplanned chronic ACSC F2 } \\
\hline & Coef. & $(95 \% \mathrm{C}) \mathrm{I}$ & $\begin{array}{l}p \\
\text { value }\end{array}$ & Coef. & $95 \% \mathrm{CI}$. & $\begin{array}{l}p \\
\text { value }\end{array}$ & Coef. & $95 \% \mathrm{CI}$ & $\begin{array}{l}p \\
\text { value }\end{array}$ \\
\hline Very high & 0.102 & $\begin{array}{l}(0.149 ; \\
0.251) \\
(0.043 ; \\
0.162)\end{array}$ & $\begin{array}{l}< \\
0.001 \\
0.001\end{array}$ & 0.623 & $\begin{array}{l}(0.441 ; \\
0.860) \\
(0.400 ; \\
0.846)\end{array}$ & $\begin{array}{l}< \\
0.001 \\
< \\
0.001\end{array}$ & 0.403 & $\begin{array}{l}(0.120 \\
0.717) \\
(0.087 \\
0.718)\end{array}$ & 0.01 \\
\hline $\begin{array}{l}\text { MMCl F1 } \\
\text { Low }\end{array}$ & REF & & & REF & & & REF & & \\
\hline Moderate & 0.007 & $\begin{array}{l}(-0.039 \\
0.053)\end{array}$ & 0.76 & 0.090 & $\begin{array}{l}(-0.092 \\
0.272)\end{array}$ & 0.33 & 0.272 & $\begin{array}{l}(-0.02 \\
0.564)\end{array}$ & 0.06 \\
\hline High & 0.002 & $\begin{array}{l}(-0.047 \\
0.051)\end{array}$ & 0.94 & 0.119 & $\begin{array}{l}(-0.065 \\
0.302)\end{array}$ & 0.20 & 0.283 & $\begin{array}{l}(-0.012 \\
0.577)\end{array}$ & 0.06 \\
\hline Very high & $\overline{0} .015$ & $\begin{array}{l}(-0.073 \\
0.043)\end{array}$ & 0.60 & 0.174 & $\begin{array}{l}(-0.024 \\
0.372)\end{array}$ & 0.08 & 0.300 & $\begin{array}{l}(-0.011 \\
0.611)\end{array}$ & 0.05 \\
\hline $\begin{array}{l}\text { MMCI baseline } \\
\text { Low }\end{array}$ & REF & & & REF & & & REF & & \\
\hline Moderate & $\overline{0} .014$ & $\begin{array}{l}(-0.051 \\
0.024)\end{array}$ & 0.46 & $\overline{0} .001$ & $\begin{array}{l}(-0.133 \\
0.131)\end{array}$ & 0.99 & 0.015 & $\begin{array}{l}(-0.187 \\
0.216)\end{array}$ & 0.88 \\
\hline High & - & $\begin{array}{l}(-0.07 \\
0.014)\end{array}$ & 0.18 & $-\overline{0}$ & $\begin{array}{l}(-0.143 \\
0.134)\end{array}$ & 0.94 & 0.052 & $\begin{array}{l}(-0.155 \\
0.258)\end{array}$ & 0.62 \\
\hline Very high & $\overline{0}-034$ & $\begin{array}{l}(-0.089 \\
0.022)\end{array}$ & 0.23 & 0.034 & $\begin{array}{l}(-0.131 \\
0.198)\end{array}$ & 0.68 & 0.006 & $\begin{array}{l}(-0.234 \\
0.246)\end{array}$ & 0.96 \\
\hline Frequency of GP contacts F2 & 0.029 & $(0.028 ; 0.03)$ & $<0.001$ & 0.016 & $\begin{array}{l}(0.014 \\
0.017)\end{array}$ & $\begin{array}{l}< \\
0.001\end{array}$ & 0.015 & $\begin{array}{l}(0.013 \\
0.017)\end{array}$ & $\begin{array}{l}< \\
0.001\end{array}$ \\
\hline Frequency of GP contacts F1 & $\overline{0} .014$ & $\begin{array}{l}(-0.015 ;- \\
0.013)\end{array}$ & $\begin{array}{l}< \\
0.001\end{array}$ & $\overline{0} .009$ & $\begin{array}{l}(-0.011 ;- \\
0.006)\end{array}$ & $\begin{array}{l}< \\
0.001\end{array}$ & $\overline{0} .007$ & $\begin{array}{l}(-0.01 ;- \\
0.004)\end{array}$ & $\begin{array}{l}< \\
0.001\end{array}$ \\
\hline Frequency of GP contacts baseline & & $\begin{array}{l}(-0.003 ;- \\
0.001)\end{array}$ & 0.001 & 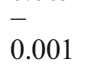 & $\begin{array}{l}(-0.003 \\
0.001)\end{array}$ & 0.38 & $\overline{0} .001$ & $\begin{array}{l}(-0.004 \\
0.002)\end{array}$ & 0.54 \\
\hline $\begin{array}{l}\text { Number of chronic disease } \\
\text { management GP contacts F2 }\end{array}$ & $-\overline{0.004}$ & $\begin{array}{l}(-0.007 \\
0.000)\end{array}$ & 0.04 & 0.026 & $\begin{array}{l}(0.017 \\
0.035)\end{array}$ & $\begin{array}{l}< \\
0.001\end{array}$ & 0.020 & $\begin{array}{l}(0.007 \\
0.032)\end{array}$ & 0.002 \\
\hline $\begin{array}{l}\text { Number of chronic disease } \\
\text { management GP contacts F1 }\end{array}$ & 0.000 & $\begin{array}{l}(-0.004 \\
0.003)\end{array}$ & 0.83 & 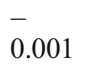 & $\begin{array}{l}(-0.011 \\
0.008)\end{array}$ & 0.77 & & $\begin{array}{l}(-0.014 \\
0.012)\end{array}$ & 0.87 \\
\hline $\begin{array}{l}\text { Number of chronic disease } \\
\text { management GP contacts baseline }\end{array}$ & $\overline{0}-001$ & $\begin{array}{l}(-0.005 \\
0.002)\end{array}$ & 0.43 & $\overline{0} .004$ & $\begin{array}{l}(-0.012 \\
0.004)\end{array}$ & 0.32 & $\overline{0} .002$ & $\begin{array}{l}(-0.012 \\
0.009)\end{array}$ & 0.75 \\
\hline Number of mental health contacts F2 & 0.001 & $\begin{array}{l}(-0.007 \\
0.01)\end{array}$ & 0.75 & $-\overline{0}-022$ & $\begin{array}{l}(-0.043 \\
0.001)\end{array}$ & 0.05 & 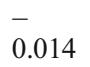 & $\begin{array}{l}(-0.027 ;- \\
0.001)\end{array}$ & 0.03 \\
\hline Number of mental health contacts F1 & 0.004 & $\begin{array}{l}(-0.006 \\
0.014)\end{array}$ & 0.46 & 0.016 & $\begin{array}{l}(-0.006 \\
0.039)\end{array}$ & 0.15 & 0.011 & $\begin{array}{l}(-0.020 \\
0.042)\end{array}$ & 0.47 \\
\hline $\begin{array}{l}\text { Number of mental health contacts } \\
\text { baseline }\end{array}$ & $\overline{0}-003$ & $\begin{array}{l}(-0.018 \\
0.012)\end{array}$ & 0.69 & $\overline{0} .009$ & $\begin{array}{l}(-0.052 \\
0.034)\end{array}$ & 0.68 & 0.005 & $\begin{array}{l}(-0.049 \\
0.06)\end{array}$ & 0.84 \\
\hline Number of specialist visits baseline & 0.001 & $\begin{array}{l}(-0.001 \\
0.002)\end{array}$ & 0.44 & 0.004 & $\begin{array}{l}(0.001 \\
0.006)\end{array}$ & 0.002 & 0.001 & $\begin{array}{l}(-0.002 \\
0.004)\end{array}$ & 0.36 \\
\hline Number of specialist visits F1 & 0.006 & $\begin{array}{l}(0.004 \\
0.007)\end{array}$ & $\begin{array}{l}< \\
0.001\end{array}$ & 0.004 & $\begin{array}{l}(0.002 \\
0.006)\end{array}$ & $\begin{array}{l}< \\
0.001\end{array}$ & 0.004 & $\begin{array}{l}(0.001 \\
0.006)\end{array}$ & 0.002 \\
\hline Unplanned hospitalisation F2_LAG1 & 0.618 & $\begin{array}{l}(0.590 \\
0.645)\end{array}$ & $\begin{array}{l}< \\
0.001\end{array}$ & & & & & & \\
\hline Unplanned hospitalisation F2_LAG2 & 0.413 & $\begin{array}{l}(0.385 \\
0.440)\end{array}$ & $<.001$ & & & & & & \\
\hline Chronic ACSC F2-LAG1 & & & & 1.145 & $\begin{array}{l}(1.030 \\
1.259)\end{array}$ & $\begin{array}{l}< \\
0.001\end{array}$ & & & \\
\hline Chronic ACSC F2-LAG2 & & & & 0.832 & $\begin{array}{l}(0.722 \\
0.943)\end{array}$ & $\begin{array}{l}< \\
0.001\end{array}$ & & & \\
\hline Unplanned ACSC F2-LAG1 & & & & & & & 1.554 & $(1.379 ; 1.73)$ & $\begin{array}{l}<.001 \\
0.001\end{array}$ \\
\hline Unplanned ACSC F2-LAG2 & & & & & & & 1.189 & $\begin{array}{l}(0.999 \\
1.378)\end{array}$ & $\begin{array}{l}< \\
0.001\end{array}$ \\
\hline Days out of hospital F2 & $\overline{0} .003$ & $\begin{array}{l}(-0.003 ;- \\
0.003)\end{array}$ & $<0.001$ & $\overline{0} .001$ & $\begin{array}{l}(-0.001 ;- \\
0.001)\end{array}$ & $\begin{array}{l}< \\
0.001\end{array}$ & $\overline{-} .001$ & $\begin{array}{l}(-0.001 ;- \\
0.001)\end{array}$ & $\begin{array}{l}< \\
0.001\end{array}$ \\
\hline
\end{tabular}

The logistic regression models were also adjusted for all baseline characteristics including sex, marital status, Indigenous status, born in Australia, education, income, SEIFA, ARIA, live independently, alcohol consumption, smoking, physical activity, sitting hours, level of limitation, psychological distress, self-report overall health, self-report quality of life, body mass index, self-report diagnosis chronic conditions, comorbidity (MACCS) at baseline and follow-up 1, Rx risk at baseline and follow-up 1

ACSC, ambulatory care sensitive condition; UPC, usual provider of care index; MMCI, Modified Modified Continuity Index; MACCS, Multipurpose Australian Comorbidity Scoring System; SEIFA, Socio-Economic Index for Areas Index of Relative Socio-economic Disadvantage; ARIA, Accessibility/ Remoteness Index of Australia; F2, the second follow-up period; F1, the first follow-up period

$*$ Wald tests for the interaction in the model: (1) unplanned hospitalisation: $\chi^{2}=1345$, $p$ value $<0.001 ;$ (2) chronic ACSC hospitalisation: $\chi^{2}=165$, $p$ value $<0.001$; and (3) unplanned chronic ACSC: $\chi^{2}=94.2, p$ value $<0.001$

$*$ Likelihood ratio tests for effect modification of multimorbidity on effect of regularity quintile: (1) unplanned hospitalisation: LR $\chi^{2}=18.5, p$ value $=$ 0.001 ; (2) chronic ACSC hospitalisation: $L R \chi^{2}=27.12$, $p$ value 0.0007; and (3) unplanned chronic ACSC: $L R \chi^{2}=21.94, p$ value $=0.005$ 
Table 4 Predictive Probability of an Individual Having Unplanned Hospitalisation, Chronic ACSC and Unplanned ACSC (Adjusted Per 1000 Persons at Risk)

\begin{tabular}{|c|c|c|c|c|c|c|c|c|c|}
\hline & \multicolumn{3}{|c|}{ Any unplanned hospitalisation } & \multicolumn{3}{|c|}{ Chronic ACSC hospitalisation } & \multicolumn{3}{|c|}{$\begin{array}{l}\text { Unplanned chronic ACSC } \\
\text { hospitalisation }\end{array}$} \\
\hline & $\begin{array}{l}\text { Point } \\
\text { estimate }\end{array}$ & $(95 \% \mathrm{CI})$ & $\begin{array}{l}p \\
\text { value }\end{array}$ & $\begin{array}{l}\text { Point } \\
\text { estimate }\end{array}$ & $(95 \% \mathrm{CI})$ & $\begin{array}{l}p \\
\text { value }\end{array}$ & $\begin{array}{l}\text { Point } \\
\text { estimate }\end{array}$ & $(95 \% \mathrm{CI})$ & $\begin{array}{l}p \\
\text { value }\end{array}$ \\
\hline \multicolumn{10}{|c|}{ A: Predictive margins (per 1000 persons at risk) } \\
\hline $\begin{array}{l}\text { Lowest } \\
\text { regularity }\end{array}$ & 287.5 & $\begin{array}{l}(283.2 \\
291.7)\end{array}$ & $\begin{array}{l}< \\
0.001\end{array}$ & 21.3 & $(19.8 ; 22.9)$ & $<$ & 11.36 & $\begin{array}{l}\text { (10.20; } \\
12.52)\end{array}$ & $<$ \\
\hline Low regularity & 267.9 & 264.5; & $\begin{array}{l}<.001 \\
0.001\end{array}$ & 20.5 & $(19.2 ; 21.8)$ & $\begin{array}{l}< \\
0.001\end{array}$ & 10.08 & $(9.15 ; 11.01)$ & $\begin{array}{l}0.001 \\
< \\
0.001\end{array}$ \\
\hline $\begin{array}{l}\text { Moderate } \\
\text { regularity }\end{array}$ & 250.2 & $\begin{array}{l}(247.0 \\
253.5)\end{array}$ & $\begin{array}{l}< \\
0.001\end{array}$ & 18.8 & $(17.6 ; 20.0)$ & $\begin{array}{l}< \\
0.001\end{array}$ & 9.36 & $(8.51 ; 10.21)$ & $\begin{array}{l}< \\
0.001\end{array}$ \\
\hline High regularity & 241.2 & $\begin{array}{l}(237.9 \\
244.5)\end{array}$ & $\begin{array}{l}< \\
0.001\end{array}$ & 19.1 & $(17.9 ; 20.2)$ & $\begin{array}{l}< \\
0.001\end{array}$ & 9.65 & $(8.82 ; 10.48)$ & $\begin{array}{l}< \\
0.001\end{array}$ \\
\hline $\begin{array}{l}\text { Highest } \\
\text { regularity }\end{array}$ & 207.5 & $\begin{array}{l}(204.0 \\
211.0)\end{array}$ & $\begin{array}{l}< \\
0.001\end{array}$ & 15.3 & $(14.2 ; 16.4)$ & $\begin{array}{l}< \\
0.001\end{array}$ & 6.68 & $(5.94 ; 7.41)$ & $\begin{array}{l}< \\
0.001\end{array}$ \\
\hline \multicolumn{10}{|c|}{ B: Contrast of the predictive margins vs. the lowest regularity (per 1000 persons at risk) } \\
\hline Low vs lowest & -19.5 & $\begin{array}{l}(-24.9 ;- \\
14.1)\end{array}$ & $\begin{array}{l}< \\
0.001\end{array}$ & -0.85 & $\begin{array}{l}(-2.86 \\
1.16)\end{array}$ & 0.40 & -1.28 & $\begin{array}{l}(-2.74 \\
0.17)\end{array}$ & 0.08 \\
\hline $\begin{array}{l}\text { Moderate vs } \\
\text { lowest }\end{array}$ & -37.2 & $\begin{array}{l}(-42.6 ;- \\
31.8)\end{array}$ & $\begin{array}{l}< \\
0.001\end{array}$ & -2.51 & $\begin{array}{l}(-4.49 ;- \\
0.54)\end{array}$ & 0.01 & -2.00 & $\begin{array}{l}(-3.45 ;- \\
0.55)\end{array}$ & 0.006 \\
\hline High vs lowest & -46.2 & $\begin{array}{l}(-51.7 ;- \\
40.7)\end{array}$ & $\begin{array}{l}< \\
0.001\end{array}$ & -2.26 & $\begin{array}{l}(-4.26 ;- \\
0.26)\end{array}$ & 0.02 & -1.71 & $\begin{array}{l}(-3.17 ;- \\
0.24)\end{array}$ & 0.02 \\
\hline $\begin{array}{l}\text { Highest vs } \\
\text { lowest }\end{array}$ & -79.9 & $\begin{array}{l}(-85.6 ;- \\
74.2)\end{array}$ & $\begin{array}{l}< \\
0.001\end{array}$ & -6.07 & $\begin{array}{l}(-8.07 ;- \\
4.08)\end{array}$ & $\begin{array}{l}< \\
0.001\end{array}$ & -4.68 & $\begin{array}{l}(-6.11 ;- \\
3.26)\end{array}$ & $\begin{array}{l}< \\
0.001\end{array}$ \\
\hline \multicolumn{10}{|c|}{ C: Contrasts of predictive margins vs. immediate lower level of regularity (per 1000 persons at risk) } \\
\hline Low vs lowest & -19.5 & $\begin{array}{l}(-24.9 ;- \\
14.1)\end{array}$ & $\begin{array}{l}< \\
0.001\end{array}$ & -0.85 & $\begin{array}{l}(-2.86 \\
1.16)\end{array}$ & 0.40 & -1.28 & $\begin{array}{l}(-2.74 \\
0.17)\end{array}$ & 0.08 \\
\hline Moderate vs low & -17.7 & $\begin{array}{l}(-22.4 ;- \\
12.9)\end{array}$ & $\begin{array}{l}< \\
0.001\end{array}$ & -1.66 & $\begin{array}{l}(-3.41 \\
0.09)\end{array}$ & 0.06 & -0.72 & $\begin{array}{l}(-1.98 \\
0.54)\end{array}$ & 0.26 \\
\hline $\begin{array}{l}\text { High vs } \\
\text { moderate }\end{array}$ & -9.0 & $\begin{array}{l}(-13.7 ;- \\
4.4)\end{array}$ & $\begin{array}{l}< \\
0.001\end{array}$ & 0.25 & $\begin{array}{l}(-1.39 \\
1.90)\end{array}$ & 0.76 & 0.29 & $\begin{array}{l}(-0.89 \\
1.47)\end{array}$ & 0.62 \\
\hline Highest vs high & -33.7 & $\begin{array}{l}(-38.4 \\
-29.0)\end{array}$ & $\begin{array}{l}< \\
0.001\end{array}$ & -3.81 & $\begin{array}{l}(-5.40 ;- \\
2.23)\end{array}$ & $\begin{array}{l}< \\
0.001\end{array}$ & -2.98 & $\begin{array}{l}(-4.07 ;- \\
1.88)\end{array}$ & $\begin{array}{l}< \\
0.001\end{array}$ \\
\hline
\end{tabular}

A: The predicted probability of an individual having the specified type of hospitalisations in F2 (adjusted for covariates in the model*1000 (i.e. per 1000 persons at risk)

B: Contrast of the predictive margins vs. the lowest regularity: differences in predictive probability of specified type of hospitalisation between higher regularity quintiles and the lowest regularity quintile

C: Contrasts of predictive margins vs. immediate lower level of regularity: differences in predictive probability of specified types of hospitalisation between the higher regularity quintiles and the immediate lower level of regularity

ACSC ambulatory care sensitive conditions, F2 the second follow-up period

a: All unplanned hospitalisations

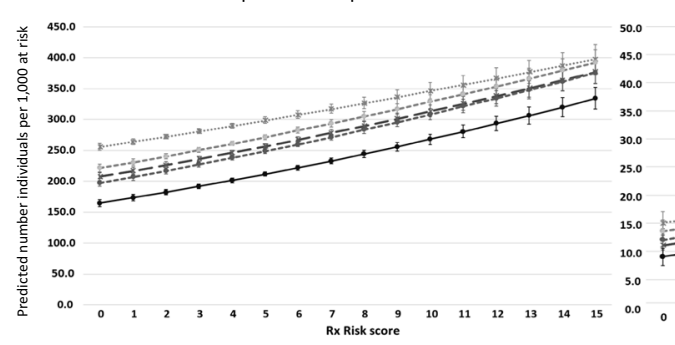

b: Chronic ACSC hospitalisations
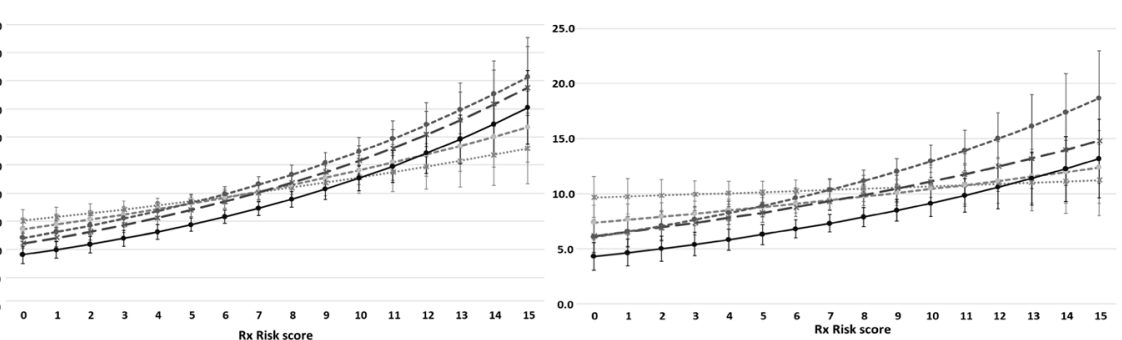

•*• Lowest regularity quintile $-\bullet-$ Low regularity quintile $-\star-$ Moderate regularity quintile $-\bullet-$ High regularity quintile $\rightarrow$ - Highest regularity quintile
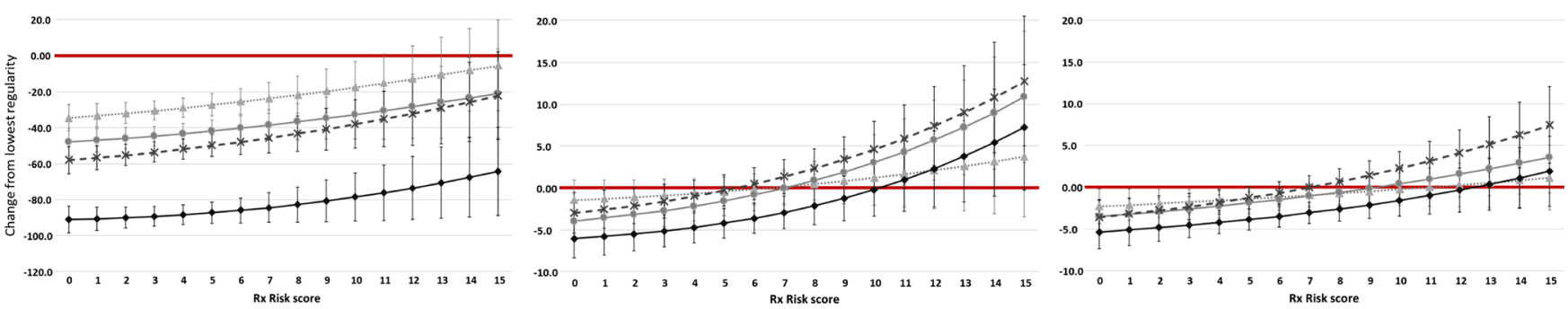

Lowest Regularity $\ldots$..... Low vs Lowest $\rightarrow$ Moderate vs Lowest $-*$ High vs Lowest $\rightarrow$ Highest vs Lowest

Fig. 1 Effect modification of multimorbidity on the relationship between unplanned hospitalisation, chronic ACSC hospitalisation and unplanned chronic ACSC hospitalisation. F1: the first follow-up period. F2: the second follow-up period. ACSC: ambulatory care sensitive condition. 
a: All unplanned hospitalisations

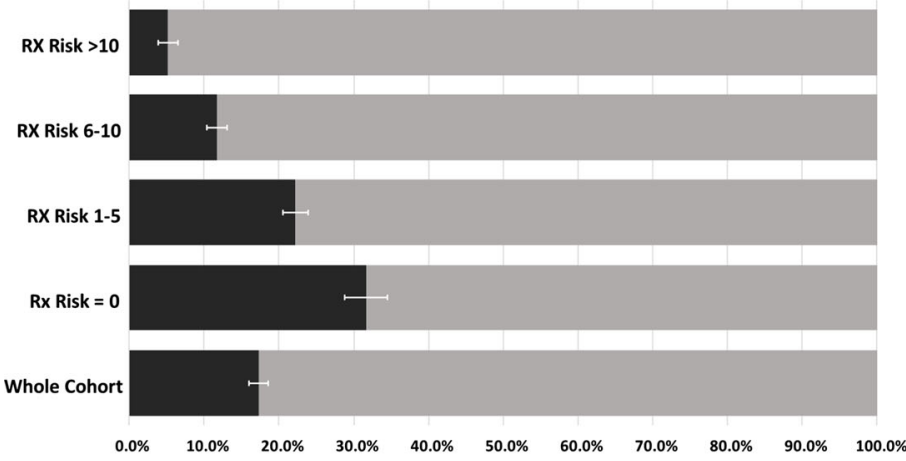

C: Unplanned chronic ACSC hospitalisations
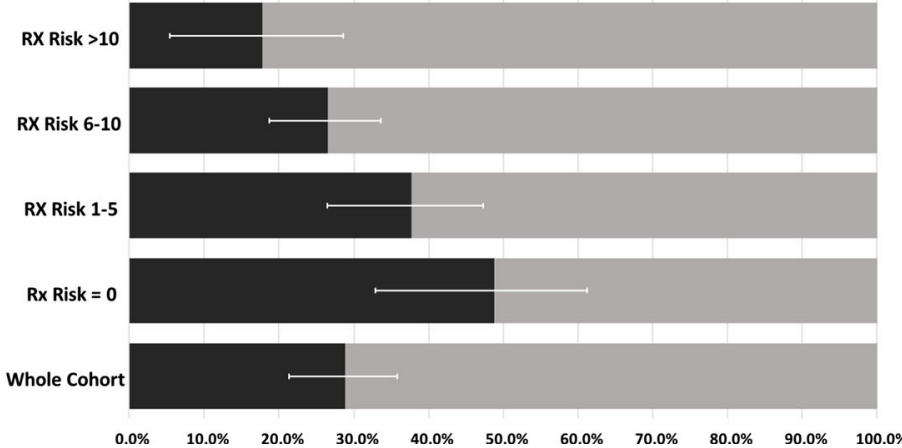

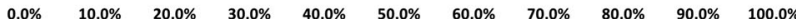

b: All chronic ACSC hospitalisations
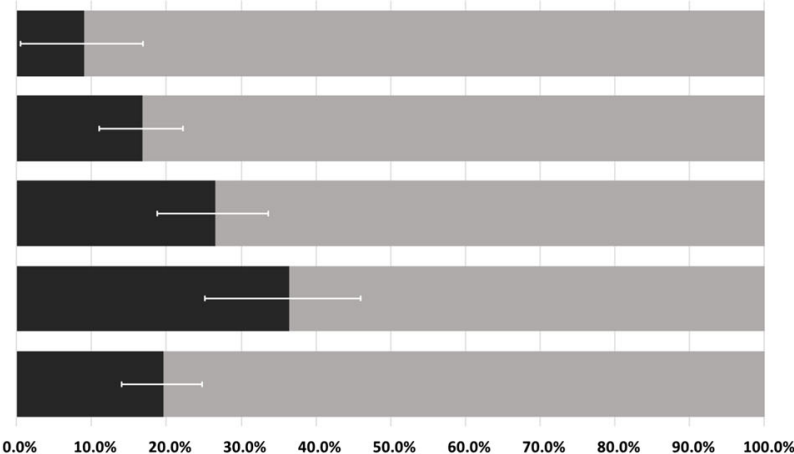

Population attributable fraction (PAF)

Population unattributable faction (PUF)

Error bars are $95 \%$ confidence intervals of the PAF

Fig. 2 Population attributable and unattributable fractions for regularity of GP contact at different level of comorbidity comparing a best case scenario (all individuals attain the highest regularity in the second follow-up period (F2)) with the world as observed in the cohort. F1: the first follow-up period. F2: the second follow-up period. ACSC: ambulatory care sensitive condition.

multimorbidity are often faced with health service challenges due to short consultation times, multiple appointments, poorly coordinated care and conflicting information between healthcare providers. ${ }^{43,}{ }^{46} \mathrm{GPs}$ report challenges in providing optimal care for people with multimorbidity as most clinical guidelines and funding models focus on single conditions ${ }^{45}$, ${ }^{47}$ in spite of a high prevalence of multimorbidity. ${ }^{48}$ GPs are often required to balance competing priorities within a limited time. ${ }^{44,}{ }^{49}$ A study in Australia found that although GPs acknowledged their role in coordinating care for patients with multimorbidity, workload pressure often limited them in fulfilling this role. ${ }^{44}$ The study also found that health professionals feel reluctant to interfere with prescribing by other health professionals, which may result in unwarranted polypharmacy ${ }^{44}$ but come at a cost of reducing the quality of care coordination across providers. Together with the evidence in the literature, our study highlights a need to foster incentives that facilitate patient-centred care (e.g. helping patients navigate between providers) and better support self-management for people with a high level of multimorbidity.

Our findings also add valuable information about the association of regularity with different specified types of hospitalisation. ACSCs are widely used as a measure of avoidable hospitalisation in evaluating performance of primary health care. ${ }^{22}$ However, the true 'preventability' of these admissions is equivocal. ${ }^{6,}{ }^{50}$ It has been argued that not all hospitalisations for ACSCs are avoidable as the count of ACSC hospitalisation depends on both population prevalence $e^{50,51}$ and the conditions defined as $\mathrm{ACSCs}^{6,52}$ that may lead to either over or undercount of avoidable hospitalisations. In our study, potentially avoidable hospitalisations were captured using not only specific chronic ACSC diagnosis codes but also a relaxed form-any unplanned hospitalisations and a restricted form-which incorporated unplanned chronic ACSCs. Notably, unplanned chronic ACSC had the highest population attributable faction to regularity of GP contacts compared with the any unplanned and chronic ACSC hospitalisations. While outside of the central study aim, these results suggest that a combination of both admission status through emergency department and diagnosis of ACSC may be a useful indicator to use in evaluating performance of primary health care.

A strength of this study is that it uses Australia's largest population-based cohort. ${ }^{19}$ The linkage of self-report and administrative data allowed for control of a wide range of confounders. Using the Rx-Risk index allows the capture of morbidity in a community-based population instead of relying on coding observed in populations with prior hospitalisation such as with the Charlson Index ${ }^{53}$ or MACSS. ${ }^{37}$ This reduces potential bias due to under-classification of multimorbidity status associated with hospital-based metrics.

The major limitation of this study is due to its crosssectional nature, though the design was chosen to address the aim of this study, which precludes assigning causality due to the uncertainty of the exposure-outcome temporal 
direction. Similarly, this study cannot identify whether regularity is a component of high quality care that has a consequential effect on the specified types of hospitalisations or whether increased regularity of care and reduction in hospitalisation are both outcomes of high quality of care. Since regularity of GP contacts was expressed in quintiles, the actual values of regularity determining each quintile are context specific. Thus, the actual values in each regularity quintile can be different between different study cohorts. In addition, the exclusion of those with less than 3 GP contacts in any follow-up period may prevent generalising the study results to the population. Finally, since the participation rate was estimated at about $18 \%$ which may cause potential biases; caution should be taken if generalising these statistics to the other population. However, a previous study suggested that the low response rate in the study cohort has a minimal effect on estimating the relationship between exposure-outcome. ${ }^{54}$

In conclusion, our study suggests that regularity of GP contact is an important consideration for designing intervention approaches to reduce avoidable hospitalisation. Given significant modification of multimorbidity on the association between regularity of GP contacts and hospitalisation, this study highlights challenges in providing optimal care for people with multimorbidity. This study shows that the association of regularity and hospitalisation weakens with increasing levels of multimorbidity; this implies that additional strategies to support primary care as a hospital avoidance strategy are warranted.

Acknowledgements: This research was completed using data collected through the 45 and Up Study (www.saxinstitute.org.au). The 45 and Up Study is managed by the Sax Institute in collaboration with major partner Cancer Council NSW; and partners: the National Heart Foundation of Australia (NSW Division); NSW Ministry of Health; NSW Government Family \& Community Services - Ageing, Carers and the Disability Council NSW; and the Australian Red Cross Blood Service. We thank the many thousands of people participating in the 45 and Up Study. We also acknowledge the Commonwealth Department of Human Services for provision of the MBS and PBS data.

Corresponding Author: Ninh Thi Ha, MHSM; Health Systems and Health Economics, School of Public Health Curtin University, Perth, Western Australia, Australia (e-mail: thi.ha@curtin.edu.au).

Funding Information This work was supported by the National Health and Medical Research Council, project grant APP1078345. The study funders had no role in the study design, conduct, manuscript writing or decision to submit for publication. Due to data access restrictions placed on the 45 and Up study data, only the approved analysts (NTH and RM) had access to the data for analysis, while all remaining authors, external and internal, had full access to all statistical reports and tables. NTH and RM can take responsibility for the integrity of the data while all authors can take responsibility for the accuracy of the data analysis.

Data Availability Statement The data that support the findings of this study are available from the relevant data custodians of the study datasets. Restrictions by the data custodians mean that the data are not publicly available or able to be provided by the authors. Researchers wishing to access the datasets used in this study should refer to the Sax Institute's 45 and Up Study process (https://www.saxinstitute. org.au/our-work/45-up-study/).
Compliance with ethical standards:

Conflict of Interest: The authors declare that they do not have a conflict of interest.

\section{REFERENCES}

1. Barnett K, Mercer SW, Norbury M, Watt G, Wyke S, Guthrie B. Epidemiology of multimorbidity and implications for health care, research, and medical education: a cross-sectional study. Lancet 2012;380(9836):37-43.

2. Cassell A, Edwards D, Harshfield A, Rhodes K, Brimicombe J, Payne $\mathbf{R}$, et al. The epidemiology of multimorbidity in primary care: a retrospective cohort study. Br J Gen Pract 2018;68(669):e245-e51.

3. Marengoni A, Angleman S, Melis R, Mangialasche F, Karp A, Garmen A, et al. Aging with multimorbidity: A systematic review of the literature. Ageing Res Rev 2011;10(4):430-9.

4. Smith SM, Soubhi H, Fortin M, Hudon C, O'Dowd T. Managing patients with multimorbidity: systematic review of interventions in primary care and community settings. BMJ 2012;345:e5205.

5. Fisher M, Baum F, Kay A, Friel S. Are changes in Australian national primary healthcare policy likely to promote or impede equity of access? A narrative review. Aust J Prim Health. 2017;23(3):209-15.

6. Gibson OR, Segal L, McDermott RA. A systematic review of evidence on the association between hospitalisation for chronic disease related ambulatory care sensitive conditions and primary health care resourcing. BMC Health Serv Res 2013;13(1):336.

7. Caminal J, Starfield B, Sanchez E. The role of primary care in preventing ambulatory care sensitive conditions. Eur $\mathrm{J}$ Pub Health 2004; 14:246-51.

8. Pollack CE, Hussey PS, Rudin RS, Fox DS, Lai J, Schneider EC. Measuring Care Continuity: A Comparison of Claims-based Methods. Med Care 2016;54(5):e30-4.

9. Pereira Gray DJ, Sidaway-Lee $K$, White E, Thorne A, Evans PH Continuity of care with doctors-a matter of life and death? A systematic review of continuity of care and mortality. BMJ Open 2018;8(6):e021161.

10. Barker I, Steventon A, Deeny SR. Association between continuity of care in general practice and hospital admissions for ambulatory care sensitive conditions: cross sectional study of routinely collected, person level data. BMJ. 2017;356:j84.

11. Youens D, Harris M, Robinson S, Preen DB, Moorin RE. Regularity of contact with GPs: Measurement approaches to improve valid associations with hospitalization. Fam Pract 2019.

12. Moorin R, Youens D, Preen DB, Wright CM. The association between continuity of provider-adjusted regularity of general practitioner contact and unplanned diabetes-related hospitalisation: A data linkage study in New South Wales, Australia using the 45 and Up Study cohort. BMJ Open 2019;9:e027158.

13. Einarsdóttir K, Preen DB, Emery JD, Holman CDAJ. Regular Primary Care Plays a Significant Role in Secondary Prevention of Ischemic Heart Disease in a Western Australian Cohort. J Gen Intern Med 2011;26(10):1092-7.

14. Einarsdóttir K, Preen DB, Emery JD, Kelman C, Holman CDAJ. Regular Primary Care Lowers Hospitalisation Risk and Mortality in Seniors with Chronic Respiratory Diseases. J Gen Intern Med 2010;25(8):766-73.

15. Youens D, Moorin R. The impact of regular General Practitioner visits on diabetic hospitalisation costs. International Health Economics Association World Congress; Boston: International Health Economics Association 2017.

16. Gibson D, Moorin R, Preen D, Emery J, Holman C. Effects of the Medicare Enahanced Primary Care program on primary care physician contact in the population of older Western Australians with chronic diseases. Aust Health Rev 2011;35:334-40.

17. Gibson D, Moorin R, Preen D, Emery J, Holman C. Enhanced Primary Care improves GP service regularity in elderly patients without impacting on service delivery. Aust J Prim Health 2012;18:295-303.

18. Benchimol EI, Smeeth L, Guttmann A, Harron K, Moher D, Petersen I, et al. The REporting of studies Conducted using Observational Routinely-collected health Data (RECORD) statement. PLoS Med 2015;12(10):e1001885.

19. Banks E, Redman S, Jorm L, Armstrong B, Bauman A, Beard J, et al. Cohort profile: the 45 and up study. Int J Epidemiol 2008;37(5):941-7. 
20. Centre for Health Record Linkage. Linking health records for research New South Wales: Centre for Health Record Linkage 2019 [Available from: http://www.cherel.org.au/. Accessed 24 April 2019.

21. Korda RJ, Du W, Day C, Page K, Macdonald PS, Banks E. Variation in readmission and mortality following hospitalisation with a diagnosis of heart failure: prospective cohort study using linked data. BMC Health Serv Res 2017;17(1):220.

22. Fleetcroft R, Hardcastle A, Steel N, Price GM, Purdy S, Lipp A, et al. Does practice analysis agree with the ambulatory care sensitive conditions' list of avoidable unplanned admissions?: a cross-sectional study in the East of England. BMJ Open. 2018;8(4).

23. Passey ME, Longman JM, Johnston JJ, Jorm L, Ewald D, Morgan GG, et al. Diagnosing Potentially Preventable Hospitalisations (DaPPHne): protocol for a mixed-methods data-linkage study. BMJ Open. 2015;5(11).

24. Australian Institute of Health and Welfare. National Healthcare Agreement: PI 18-Selected potentially preventable hospitalisations, 2015 Canberra, Australian Capital Territory, Australia2015 [Available from: https://meteor.aihw.gov.au/content/index.phtml/itemId/559032. Accessed 8 Feb 2019.

25. Australian Institute of Health and Welfare. Australian hospital statistics 2011-12. Health services series no. 50. Cat. no. HSE 134. . Canberra, Australia Australian Institute of Health and Welfare, ; 2013.

26. Australian Government DoH. MBS online Canberra: Australian Government, Department of Health; 2018 [fore relevant year, last updated 2018]. Available from: http://www.health.gov.au/internet/mbsonline/publishing.nsf/Content/Medicare-Benefits-Schedule-MBS-1. Accessed 24 Sep 2018.

27. Direct $\mathbf{H}$. The role of a GP: Healthdirect; 2018 [Available from: https:// www.healthdirect.gov.au/the-role-of-a-gp. Accessed 18 Dec 2019.

28. Pratt NL, Kerr M, Barratt JD, Kemp-Casey A, Kalisch Ellett LM, Ramsay E, et al. The validity of the Rx-Risk Comorbidity Index using medicines mapped to the Anatomical Therapeutic Chemical (ATC) Classification System. BMJ Open 2018;8(4):e021122.

29. Magill MK, Senf J. A new method for measuring continuity of care in family practice residencies. J Fam Pract 1987;24(2):165-8.

30. Breslau N, Haug MR. Service delivery structure and continuity of care: a case study of a pediatric practice in process of reorganization. J Health Soc Behav 1976;17(4):339-52

31. Australian Institute of Health and Welfare. The Active Australia Survey: a guide and manual for implementation, analysis and reporting. . Canberra, Australia. : Australian Institute of Health and Welfare,; 2003.

32. Kessler RC, Andrews G, Colpe LJ, Hiripi E, Mroczek DK, Normand SL, et al. Short screening scales to monitor population prevalences and trends in non-specific psychological distress. Psychol Med 2002;32(6):959-76.

33. Rand Healthcare. 36-Item Short Form Survey Instrument (SF-36) Santa Monica, California, United States Rand Healthcare, ; 2019 [Available from: https://www.rand.org/health-care/surveys_tools/mos/36-itemshort-form/survey-instrument.html. Accessed 22 Feb 2019.

34. Koenig HG, Westlund RE, George LK, Hughes DC, Blazer DG, Hybels C. Abbreviating the Duke Social Support Index for use in chronically ill elderly individuals. Psychosomatics. 1993;34(1):61-9.

35. Australian Bureau of Statistics. Census of population housing: Socioeconomic indexes for areas. Canberra: ABS; 2006, 2011.

36. Australian Bureau of Statistics. ABS Maps (Remoteness Structure). Canberra, Australia. : Australian Bureau of Statistics, ; 2006, 2011 [8 June 2018 ]. Available from: http://stat.abs.gov.au/itt/r.jsp?ABSMaps
37. Holman C, Preen D, Baynham N, Finn J, Semmens J. A multipurpose Australian comorbidity scoring system performed better than the Charlson index J Clin Epidemiol 2005;58:1006-14.

38. Statacorp. Test linear hypotheses after estimation [Available from: https://www.stata.com/manuals 13/rtest.pdf. Accessed 20 Sep 2019.

39. Royston P, Sauerbrei W. Two techniques for investigating interactions between treatment and continuous covariates in clinical trials. Stata $\mathrm{J}$ 2009;9:230-51.

40. Newson RB. Attributable and unattributable risks and fractions and other scenario comparisons. . Stata J 2013;13:672-98.

41. Statacorp. Stata statistical software: release 16. . College Station, Texas, United States2019.

42. Moorin RE, editor. Measuring the influence of regularity of GP contact on diabetic potentially preventable hospitalisations. Health Services \& Policy Research Conference; 2015; Melbourne.

43. Millar E, Stanley J, Gurney J, Stairmand J, Davies C, Semper K, et al. Effect of multimorbidity on health service utilisation and health care experiences. J Prim Health Care 2018;10(1):44-53.

44. Mc Namara KP, Bell JS, Breken BD, Alzubaidi HT, Dunbar JA, Walker C, et al. Health professional perspectives on the management of multimorbidity and polypharmacy for older patients in Australia. Age Ageing 2016;46(2):291-9.

45. Sinnott C, Mc Hugh S, Browne J, Bradley C. GPs' perspectives on the management of patients with multimorbidity: systematic review and synthesis of qualitative research. BMJ Open 2013;3(9):e003610.

46. Burgers JS, Voerman GE, Grol R, Faber MJ, Schneider EC. Quality and Coordination of Care for Patients With Multiple Conditions: Results From an International Survey of Patient Experience. Eval Health Prof 2010;33(3):343-64.

47. Guthrie B, Payne K, Alderson P, McMurdo MET, Mercer SW. Adapting clinical guidelines to take account of multimorbidity. BMJ 2012;345:e6341.

48. Salisbury C, Johnson L, Purdy S, Valderas JM, Montgomery AA. Epidemiology and impact of multimorbidity in primary care: a retrospective cohort study. Br J Gen Pract 2011;61(582):e12-e21.

49. Stokes T, Tumilty E, Doolan-Noble F, Gauld R. Multimorbidity, clinical decision making and health care delivery in New Zealand Primary care: a qualitative study. BMC Fam Pract. 2017;18(1):51.

50. Walker RL, Ghali WA, Chen G, Khalsa TK, Mangat BK, Campbell NRC, et al. ACSC Indicator: testing reliability for hypertension. BMC Med Inform Decis Mak. 2017;17(1):90.

51. Rizza P, Bianco A, Pavia M, Angelillo IF. Preventable hospitalization and access to primary health care in an area of Southern Italy. BMC Health Serv Res. 2007;7:134.

52. Purdy S, Griffin T, Salisbury C, Sharp D. Ambulatory care sensitive conditions: terminology and disease coding need to be more specific to aid policy makers and clinicians. Public Health 2009;123(2): 169-73.

53. Charlson M, Szatrowski T, Peterson J, Gold J. Validation of a combined comorbidity index. J Clin Epidemiol 1994;47:1245-51.

54. Mealing NM, Banks E, Jorm LR, Steel DG, Clements MS, Rogers KD. Investigation of relative risk estimates from studies of the same population with contrasting response rates and designs. BMC Med Res Methodol 2010;10:26.

Publisher's Note Springer Nature remains neutral with regard to jurisdictional claims in published maps and institutional affiliations. 